\title{
The Impact of Collegiate Outcomes of Learning Assessment and Academic Performance on University Graduates' Employment
}

\author{
Sih-Tong Wu \\ National Sun Yat- sen University/Education
}

\begin{abstract}
Recent research on self-regulated learning has showed student regulated their learning of effort by using learning strategies and learning motivation. Much of this research has explored these factors without consideration of the prediction job performance. The main purpose of this paper is to explore the effects of learning strategies, learning motivation, academic performance on their graduates' employment. The participants comprised after one-year graduated of National Sun Yat-sen University 2500 students. The analyzing Collegiate Outcomes of Learning Assessment (COLA) System that the survey of junior students of 105 original academic data and survey of after one-year graduated 106 employment condition. Statistical analysis was conducted through descriptive statistic, one-way ANOVA and Chi-square. Results were as follow: learning strategies on academic performance, learning motivation on academic performance, academic performance on graduates' employment are evident. According to the analysis result, we learned that academic performance affects work performance while learning motivation and learning strategies are intervening variables. Therefore, educators should promote the motivation and strategy of students. In future research, it is recommended to add more qualitative interviews.
\end{abstract}

Keywords: learning strategies, learning motivation, Collegiate Outcomes of Learning Assessment System, National Sun Yat-sen University, ANOVA, Chi-square Test 